\title{
Prenatal maternal transdiagnostic, RDoC-informed predictors of newborn neurobehavior: Differences by sex
}

\author{
Mengyu (Miranda) $\mathrm{Gao}^{1} \mathbb{D}$, Brendan Ostlund², Mindy A. Brown ${ }^{1}$, Parisa R. Kaliush ${ }^{1}$, Sarah Terrell², \\ Robert D. Vlisides-Henry ${ }^{1}$, K. Lee Raby ${ }^{1}\left(\mathbb{D}\right.$, Sheila E. Crowell ${ }^{1}(\mathbb{D})$ and Elisabeth Conradt ${ }^{1}$ \\ ${ }^{1}$ Department of Psychology, University of Utah, Salt Lake City, UT, USA and ${ }^{2}$ Department of Psychology, Pennsylvania State University, University Park, PA, USA
}

\begin{abstract}
We examined whether Research Domain Criteria (RDoC)-informed measures of prenatal stress predicted newborn neurobehavior and whether these effects differed by newborn sex. Multilevel, prenatal markers of prenatal stress were obtained from 162 pregnant women. Markers of the Negative Valence System included physiological functioning (respiratory sinus arrhythmia [RSA] and electrodermal [EDA] reactivity to a speech task, hair cortisol), self-reported stress (state anxiety, pregnancy-specific anxiety, daily stress, childhood trauma, economic hardship, and family resources), and interviewer-rated stress (episodic stress, chronic stress). Markers of the Arousal/Regulatory System included physiological functioning (baseline RSA, RSA, and EDA responses to infant cries) and self-reported affect intensity, urgency, emotion regulation strategies, and dispositional mindfulness. Newborns' arousal and attention were assessed via the Neonatal Intensive Care Unit (NICU) Network Neurobehavioral Scale. Path analyses showed that high maternal episodic and daily stress, low economic hardship, few emotion regulation strategies, and high baseline RSA predicted female newborns' low attention; maternal mindfulness predicted female newborns' high arousal. As for male newborns, high episodic stress predicted low arousal, and high pregnancy-specific anxiety predicted high attention. Findings suggest that RDoC-informed markers of prenatal stress could aid detection of variance in newborn neurobehavioral outcomes within hours after birth. Implications for intergenerational transmission of risk for psychopathology are discussed.
\end{abstract}

Keywords: neurobehavior, prenatal, RDoC, sex differences, transdiagnostic

(Received 8 December 2020; revised 20 December 2020; accepted 22 December 2020)

Identifying prenatal factors that may confer susceptibility for problematic offspring outcomes has far-reaching health and social implications. However, a significant challenge for research in this area is capturing the complexity of prenatal risk factors. Focusing on a single vulnerability factor fails to appreciate the full range of pregnant women's emotional, cognitive, and physiological experiences (Doyle \& Cicchetti, 2018). The National Institute of Mental Health's Research Domain Criteria (RDoC) initiative was introduced to advance understanding of psychopathology based on dimensions that transcend diagnostic boundaries (Insel et al., 2010), which can be contrasted with the highly specified categories in the fifth edition of Diagnostic and Statistical Manual of Mental Disorders (DSM-5). By encompassing multiple domains of functioning, the $\mathrm{RDoC}$ may provide a more comprehensive framework to begin unraveling the complexity of prenatal maternal stress (Doyle \& Cicchetti, 2018).

Author for Correspondence: Mengyu (Miranda) Gao, Ph.D., Department of Psychology, University of Utah, 380 South 1530 East, Salt Lake City, UT 84112; E-mail: m.gao@psych.utah.edu

Cite this article: Gao M(Miranda), Ostlund B, Brown MA, Kaliush PR, Terrell S Vlisides-Henry RD, Raby KL, Crowell SE, Conradt E (2021). Prenatal maternal transdiagnostic, RDoC-informed predictors of newborn neurobehavior: Differences by sex. Development and Psychopathology 33, 1554-1565. https://doi.org/10.1017/ S0954579420002266
The RDoC approach is well suited for studies of the intergenerational consequences of prenatal stress for two reasons. First, RDoC's hallmark feature of multiple units of analysis (e.g., molecules, cells, circuits, physiology, and behavior; Insel et al., 2010) can guide studies of the potentially multiple pathways by which prenatal risk impact children's outcomes. Second, RDoC's central goal of understanding neurodevelopmental origins of psychopathology necessarily requires a focus on early development, which in turn may help inform targeted preventive intervention efforts (Insel et al., 2010; Mittal \& Wakschlag, 2017).Prenatal stress is a multifaceted concept and may be characterized as an emergent property of multiple RDoC domains. In the current study, we focused on two RDoC domains - the Negative Valence and Arousal/Regulatory Systems. We examined whether markers of susceptibility under each domain predicted individual differences in newborn neurobehavior and whether there were differences by newborn sex.

\section{Prenatal stress in RDoC: The negative valence systems}

In a large-scale community study, an estimated $30 \%$ of pregnant women reported some type of stress in their daily lives, including anxiety and depressive symptoms (Loomans et al., 2013). High levels of sadness and anxiety experienced prenatally are predictive of impaired newborn neurobehavior, dysregulated infant 
Table 1. Summary of Research Domain Criteria (RDoC)-informed predictors under each domain, with corresponding measures and hypothesized effect

\begin{tabular}{|c|c|c|c|}
\hline $\begin{array}{l}\text { RDoC } \\
\text { domains }\end{array}$ & Proposed RDoC-informed predictors & Measures & $\begin{array}{l}\text { Hypothesized effect- Newborn's high arousal } \\
\text { and low attention will be predicted by: }\end{array}$ \\
\hline \multicolumn{4}{|c|}{ Negative valence system } \\
\hline \multicolumn{4}{|c|}{ - Acute threat } \\
\hline & 1. EDA reactivity to acute threat & a1) EDA reactivity (to TSST speech task) & - high EDA reactivity \\
\hline & 2. RSA reactivity to acute threat & b1) RSA reactivity (to TSST speech task) & - Iow RSA reactivity \\
\hline & $\begin{array}{l}\text { 3. Number of episodic stress } \\
\text { experienced in the last } 6 \text { months }\end{array}$ & c) UCLA Life Stress Interview & - more episodic stress \\
\hline \multicolumn{4}{|c|}{ - Potential threat } \\
\hline & 4. EDA reactivity to potential threat & a2) EDA reactivity (during TSST preparation) & - high EDA reactivity \\
\hline & 5. RSA reactivity to potential threat & b2) RSA reactivity (during TSST preparation) & - Iow RSA reactivity \\
\hline & 6. State anxiety & d) State-Trait Anxiety Inventory - State & - high state anxiety \\
\hline & 7. Pregnancy-specific anxiety & e) Pregnancy-Specific Anxiety & - high pregnancy-specific anxiety \\
\hline \multicolumn{4}{|c|}{ - Sustained threat } \\
\hline & 8. Maltreatment history & f) Childhood Trauma Quest ionnaire & - high levels of childhood trauma \\
\hline & 9. Dysregulated HPA axis & g) Hair cortisol & - high levels of hair cortisol concentrations \\
\hline & 10. Economic hardship & h) Economic Hardship & - high economic hardship \\
\hline & $\begin{array}{l}\text { 11. Chronic stress experienced in the } \\
\text { past } 6 \text { months }\end{array}$ & c) UCLA Life Stress Interview & - high levels of chronic stress \\
\hline & 12. Family resources & i) Family Resources & - low family resources \\
\hline & 13. Day-to-day stress & j) Everyday Stress Index & - high levels of everyday stress \\
\hline \multicolumn{4}{|c|}{ Arousal/regulatory system } \\
\hline \multicolumn{4}{|l|}{ - Arousal } \\
\hline & 14. EDA reactivity to infant cry & a3) EDA reactivity (to infant cry) & - High EDA reactivity \\
\hline & 15. RSA reactivity to infant cry & b3) RSA reactivity (to infant cry) & - Low RSA reactivity \\
\hline & 16. Affect intensity & k) Affect Intensity Measure & - High affect intensity \\
\hline & 17. Impulsivity & $\begin{array}{l}\text { l) Urgency, Premeditation, Perseverance, } \\
\text { Sensation seeking, Impulsive behavior scale }\end{array}$ & - High levels of impulsivity \\
\hline \multicolumn{4}{|c|}{ - Regulation } \\
\hline & 18. RSA activity at rest (baseline) & b4) RSA activity (TSST baseline) & - Low baseline RSA \\
\hline & 19. Mindfulness & $\begin{array}{l}\text { m) The Mindful Attention and Awareness } \\
\text { Scale }\end{array}$ & - Low levels of mindfulness \\
\hline & $\begin{array}{l}\text { 20. Difficulties in accessing effective } \\
\text { emotion regulation strategies }\end{array}$ & n) DERS- strategy subscale & - High difficulty of accessing strategies \\
\hline
\end{tabular}

DERS = Difficulties in Emotion Dysregulation Scale; EDA = electrodermal reactivity; HPA = hypothalamus-pituitary-adrenal; RSA = respiratory sinus arrhythmia; TSST = Trier Social Stress Test

behavior, and higher risk for adolescent mental disorder (Goodman \& Gotlib, 1999; O’Donnell, Glover, Barker, \& O'Connor, 2014). Therefore, anxiety, depression, and fear in pregnant women are particularly relevant for studies of the effects of prenatal stress on offspring outcomes. Within the RDoC framework, the Negative Valence Systems include constructs such as sustained threat, potential threat ("anxiety"), and acute threat ("fear"). Consistent with RDoC's approach of multiple levels of analysis, we identified multiple indicators of prenatal stress for each Negative Valence Systems construct (see Table 1).

\section{Sustained threat}

Pregnant women experienced different levels of preexisting, prolonged exposure to a wide range of stressors. Stress associated with socioeconomic disadvantages, for instance, can affect fetal development and has been linked to low birth weight (Bryant Borders, Grobman, Amsden, \& Holl, 2007). In addition to ongoing life stress, early life adversity may negatively affect fetal and newborn health (Buss et al., 2017). A possible biomarker of prenatal chronic stress is maternal hair cortisol, which has been associated with prenatal stress and anxiety (Orta et al., 2019) as well as alterations in infants' neurobiological functioning (Wachman et al., 2019).

\section{Potential threat}

Sustained and generalized stress across the life span may be compounded by the uncertainty that is unique to pregnancy. For some women, the anticipated birth of a child may incur unknown, 
potential threats, including concerns about the health of the fetus, uncertainty surrounding labor and delivery, and fear of medical complications (Dunkel Schetter, 2011). Pregnancy-specific anxieties may prepare an expectant mother for forthcoming, unknown risks associated with birth and childrearing, thereby serving as an adaptive alert response system to potential threat. An exaggerated threat state, however, may confer vulnerability to the developing fetus by modulating uteroplacental hormonal concentrations and blood flow (Dipietro, Ghera, \& Costigan, 2008).

Markers of potential threat during pregnancy also exist on the physiological level. Respiratory sinus arrhythmia (RSA) and electrodermal activity (EDA), respective measures of the parasympathetic and sympathetic nervous systems, work in concert to prepare an expectant woman's body for environmental demands. RSA - a measure of heart rate variability across respiration - is thought to index parasympathetic (i.e., inhibitory) influences on cardiac output (Beauchaine, 2015). In the face of environmental challenges, the parasympathetic nervous system withdraws its inhibitory influence on the heart, resulting in faster heart rate and less beat-to-beat variability (i.e., decreased RSA) to help the body shift attentional and behavioral resources. Although modest reductions in RSA reflect a healthy and appropriate response to challenge, exaggerated or blunted RSA reactivity may indicate problematic attempts at physiological regulation (Beauchaine, 2015). Sympathetic nervous system reactivity can be measured by activation of eccrine sweat glands, which yields increased sweat production (i.e., increased EDA). EDA has been theorized to underlie emotions, stress, and coping, and reduced EDA in anticipation of adverse events is related to risk for psychopathology (Sarchiapone et al., 2018).

\section{Acute threat}

Another aspect of prenatal stress may be captured by how pregnant women respond to acute threats (i.e., threat stimuli to which individuals are acutely exposed). Physiological and behavioral responses to acute environmental challenges, such as episodic life events, may provide a glimpse into individuals' regulatory abilities and portend psychopathology (Beauchaine, 2015). Women who experience high levels of anxiety exhibit smaller decreases in RSA in response to acute stressors compared to those who are less anxious (Braeken et al., 2015). These smaller decreases in RSA may reflect diminished control of the parasympathetic nervous system, which confers risk for problematic parenting behaviors and newborn neurobehaviors (Ostlund et al., 2019). The number of stressful life events experienced during pregnancy is also a critical component of prenatal stress; the number of stressful life events during pregnancy predicts child outcomes, even with stringent statistical controls (Robinson et al., 2011).

\section{Prenatal stress in RDoC: The arousal/regulatory systems}

Within the RDoC framework, the Arousal/Regulatory Systems involve the activation of neural systems for context-appropriate responses and the regulation of these neural systems by energy balance and sleep (Kozak \& Cuthbert, 2016). A range of markers, such as RSA and impulsivity/hyperactivity, are employed to measure the Arousal/Regulatory Systems among nonpregnant adults (Arns, Swatzyna, Gunkelman, \& Olbrich, 2015).Arousal and regulation are two useful constructs in this domain to better understand prenatal maternal stress (see Table 1).

\section{Arousal}

Arousal is thought to reflect individual differences in sensitivity to external and internal stimuli. Women high in arousal are more likely to experience intense affect and exhibit impulsive and dysregulated behaviors than women who are low in arousal. For instance, among a sample of pregnant women assessed during their third trimester, emotion dysregulation differentiated women who endorsed a prior lifetime history of self-injury from those who did not (Lin et al., 2019). Another marker of arousal among pregnant women is their RSA and EDA reactivity to infant cries. Although a highly aroused state is often regarded as a sign of poor regulation (Porges, 2007), it may be adaptive in the formation stages of caregiver-offspring relationships, because it may facilitate caregivers' behavioral responses to infants' needs, such as increased parental sensitivity (Ablow, Marks, Feldman, \& Huffman, 2013).

\section{Regulation}

Although enhanced arousal may increase alertness to potential environmental threats, dampened arousal may benefit maintenance of homeostasis. Appropriate and flexible levels of arousal allow individuals to interact with the environment in a contextspecific manner (Kozak \& Cuthbert, 2016).In the current study, we expanded upon the existing definition of the Arousal/ Regulatory System-informed construct regulation to capture how individuals modulate arousal levels to cope with environmental stress. Although regulation is not specified in the current RDoC matrix as a distinct construct, regulatory abilities are fundamental for modulating neural and physiological systems to meet environmental demands. In addition to endogenous physiological oscillations, such as sleep, individuals can modulate arousal by exogenous behavioral processes, such as affect regulation (Kozak \& Cuthbert, 2016). In this study, we identified two behavioral markers of regulation (see Table 1). First, women with limited access to emotion regulation strategies are more likely to experience difficulties in regulating emotions (Gratz \& Roemer, 2004).Second, greater maternal mindfulness is related to less perinatal anxiety, less negative affectivity, and more self-regulatory behaviors in infants (van den Heuvel, Johannes, Henrichs, \& Van den Bergh, 2015). Finally, baseline RSA is a marker of individuals' capacity for physiological regulation, and low baseline RSA has been linked to various measures of mental health in pregnant women, such as self-injurious thoughts and behaviors and depression (Lin et al., 2019).

\section{Newborn neurobehavior}

Establishing early signs of problematic development using the RDoC framework could facilitate early identification of populations that are vulnerable to the development of psychopathology. Evident from the first hours after birth, newborn neurobehavioral competencies are a set of observable behavioral outcomes that emerge as a result of the dynamic interplay between neurophysiological, cognitive, and motor processes (Monk \& Hane, 2014). Newborn neurobehavior is thought to be sensitive to a range of prenatal influences and may lay the foundation for later emotional and behavioral development (Lester \& Tronick, 2004; Monk \& Hane, 2014). These competencies can be measured via the Neonatal Intensive Care Unit (NICU) Network Neurobehavioral Scale (NNNS), a standardized protocol for reliable assessment of low- and high-risk neonates (Lester \& Tronick, 2004). The NNNS captures a variety of functional domains in newborns, 
such as responses to stimuli (i.e., gaze, sustained alertness), irritability and fussiness, and ability to be soothed. A recent study on newborn neurobehavior identified two factors, attention and arousal, from the various NNNS dimension scores (Ostlund et al., 2019). Low attention and high arousal may be early signs of newborn difficulties, although findings have been mixed. Specifically, infants who scored low on attention exhibited difficulty with attention deployment, such as lack of engagement with the environment, inability to engage with auditory and visual stimulation while regulating their behavioral state, and interrupted alertness and discordant visual tracking. Infants who scored high on arousal were highly sensitive to the environment, exhibiting low thresholds for stimulation and high proneness to excitability (i.e., fussiness; Lester, Tronick, \& Brazelton, 2004; Ostlund et al., 2019).

The factor names, attention and arousal, reflected their respective resemblance to the "attention" and "arousal" constructs in RDoC. These two factors were predicted by prenatal exposure to maternal emotion dysregulation (Ostlund et al., 2019), a transdiagnostic marker of psychopathology that lies at the intersection of RDoC domains (Fernandez, Jazaieri, \& Gross, 2016). Therefore, the NNNS lends itself to studying the effect of $\mathrm{RDoC}$-informed prenatal stress as a critical marker of newborn neurobehavioral functioning.

\section{Sex differences}

Male fetuses may be more susceptible to prenatal insults than female fetuses; however, mechanisms underlying male vulnerability are largely unclear (Dipietro \& Voegtline, 2017; Spinillo et al., 2009; Willoughby, Greenberg, Blair, \& Stifter, 2007). Further, little is known about whether prenatal maternal predictors of newborn neurobehavior differ as a function of newborn sex. Without assessment of neurobehavior at birth, it is unclear whether male vulnerability to prenatal influences that have been observed in infancy and early childhood reflects the persistent effect of prenatal risk on male offspring or as the result of an interplay between prenatal and postnatal experiences.

\section{Current study}

The present study had two aims. First, we examined whether constructs related to prenatal stress that are from two RDoC domains - Negative Valence Systems and Arousal/Regulatory Systems - predicted newborn neurobehavior. For the Negative Valence Systems, we hypothesized that markers of greater maternal acute, potential, and sustained threat would predict high newborn arousal and low attention. For the Arousal/Regulatory Systems, we hypothesized that markers of high arousal and low regulation would predict high newborn arousal and low attention. Our second aim was to examine whether these associations would differ as a function of newborn sex. We hypothesized that the associations between the markers of prenatal stress and poor newborn functioning would be stronger for male than for female newborns. Aims, hypotheses, and the analytic plan were preregistered on the Open Science Framework (osf.io/m745d).

\section{Method}

\section{Participants}

Pregnant women were recruited from obstetrics and gynecology clinics affiliated with the University of Utah and local communities through flyers, brochures, advertisements, and postings on social media. Eligibility criteria included: fluent in English or Spanish, 18-40 years of age, 25 weeks or more into pregnancy, no pregnancy complications at the time of recruitment (e.g., preeclampsia, gestational diabetes), no substance use during pregnancy, anticipated singleton delivery, and planned delivery at a participating hospital. We oversampled women with high and low levels of emotion dysregulation in an attempt to achieve a uniform distribution of scores on the Difficulties in Emotion Regulation Scale (DERS; Gratz \& Roemer, 2004). See Lin et al. (2019) for additional recruitment information.

The final sample included 162 pregnant women who were on average 29 years old (range 18-40). The majority of women (79\%) self-identified as White (25.3\% Hispanic White), 9.3\% as Asian, $6.1 \%$ as multiracial, and the rest (5.5\%) as American Indian/ Alaskan Native, Hawaiian Native/Pacific Islander, or Black/ African American. Most participants had high levels of formal education (63\% attended college, $19.1 \%$ attended graduate school, $13 \%$ were high school graduate or equivalent, and $3.1 \%$ did not finish 12th grade). Most women were married or lived with a romantic partner (91.4\% cohabitating; $75.9 \%$ married). The median annual household income was in the $\$ 50,000-\$ 79,999$ range.

Newborn neurobehavior was assessed between 1 day and 20 days after delivery $\left(M_{\text {days }}=2.3, M d n_{\text {days }}=1.0, S D=3.2\right.$, range: 1-20 days). Six newborns were assessed outside of above time window (range $=28-59$ days) due to scheduling difficulties. One hundred fifty-five newborn infants were examined (77 males, 47.5\%). Among the seven newborns whose data were not available, three mothers declined the assessment at the hospital, one mother withdrew from the study, one mother experienced a fetal demise, one mother was incarcerated, and one mother was unable to be reached. Most deliveries were vaginal (72.8\%). Average gestational age at time of delivery was 275 days (range $=$ 239-290 days). Based on mothers' reports, $73.5 \%$ of the infants were White (24.1\% Hispanic White), $21 \%$ were multiracial $(5.6 \%$ Hispanic multiracial), $2.5 \%$ were Asian, and the remainder were Black/African American, American Indian/Alaskan Native, or Hawaiian/Pacific Islander.

\section{Procedures}

After completing online questionnaires, participants visited our research laboratory between the 26th and 40th weeks of pregnancy to complete a series of tasks, including the Trier Social Stress Test (TSST; Kirschbaum, Pirke, \& Hellhammer, 1993) and a recorded infant cry task. Research assistants placed sensors on participants to record their autonomic functioning. Following a 10-min baseline period, participants were given $3 \mathrm{~min}$ to prepare a speech for a hypothetical interview. They were then instructed to deliver the speech in front of a camera for $5 \mathrm{~min}$ while experimenters pretended to evaluate their performances. Following a 10-min recovery period, the infant cry task was administered during which participants watched a series of 60-s video clips. A clip of a baby playing, followed by a clip of a baby crying, was presented in between two clips of neutral landscapes. After this task, participants completed a series of semistructured interviews, including the UCLA Life Stress Interview (Hammen et al., 1987). At the end of the prenatal visit, participants were debriefed and compensated. Participants and their newborn infants were visited at the hospital post-delivery when possible. A trained examiner administered the NNNS, which 
was scored upon conclusion of the exam. All participants provided written consent before each phase of the study. Our protocol was approved by the University's Institutional Review Board.

\section{Measures}

EDA reactivity (Table 1, a1, a2, a3)

Mothers' EDA was recorded during TSST task and during infant cry task at the prenatal visit. EDA signals were recorded with two rectangular spot electrodes on the plantar surface of hand(van Dooren, de Vries, \& Janssen, 2012), using BioLab acquisition software (version 3.1) and MindWare wireless mobile devices (MindWare Technologies Ltd., Gahanna, OH). An impedance cardiograph signal was simultaneously recorded and was later used to derive participants' respiration pattern. Both signal sources were examined, along with synchronized video footage of the data collection session, by trained research assistants for scoring EDA data. Specifically, the number of fluctuations in the skin conductance greater or equal to 0.05 microsiemens was identified and recorded (Dawson, Schell, \& Filion, 2007) in 30-s epochs. Research assistants watched for and removed responses corresponding to situations that might lead to artifacts (e.g., equipment failure). A senior researcher reviewed data for ambiguous cases, and outliers were examined using box and whisker plots upon completion of all scoring.

Three sets of EDA reactivity scores were calculated. EDA reactivity to TSST speech task was calculated by subtracting the mean number of EDA responses during 10-min baseline $\left(\mathrm{EDA}_{\text {baseline }}\right)$ from the 5-min speech task of TSST. Similarly, EDA reactivity during TSST preparation was calculated by subtracting $\mathrm{EDA}_{\text {baseline }}$ from participants' mean EDA during the preparation phase of the TSST. Lastly, mothers' EDA reactivity to infant cry was calculated by subtracting the mean number of responses during the first 60-s of neutral landscape $\left(\mathrm{EDA}_{\text {first_landscape }}\right)$ from their EDA responses during infant cry task. Higher EDA reactivity scores indicated greater sympathetic system arousal.

\section{RSA baseline and reactivity (Table 1, b1, b2, b3, b4)}

Mothers' RSA was recorded during TSST task and during infant cry task at the prenatal visit. Trained research assistants screened and scored the data in 30-s epochs. A senior investigator reviewed, rescored, and finally determined whether unusually high/low values (i.e., values below 2 or above 10) should be retained. RSA reactivity scores were derived similarly as the EDA scores.

\section{Episodic and chronic stress (Table 1, C)}

UCLA Life Stress Interview (Hammen et al., 1987) - a semistructured interview - was used to assess participants' episodic and chronic stress in the past 6 months. Trained interviewers evaluated multiple domains of the participants' life (including close friendships, relationship with partner, co-parenting with baby's father, dating, relationship with family, finances, work status, neighborhood environment, school, and health) on a 5-point scale $(1-$ low stress to 5 - high stress). An average score from all life domains was derived for each participant to indicate chronic stress. Participants also reported acute life events (or episodic stressors) and were asked to rate how severe the threat or impact each event was on a 5-point scale ( $1-$ no/minimal impact to 5 - severe/catastrophic impact). Total number of events that received subjective rating of at least 3 was used to indicate participants' episodic stress.

\section{State anxiety (Table 1, d)}

The State-Trait Anxiety Inventory - State (STAI-S; Spielberger, Gorsuch, Lushene, Vagg, \& Jacobs, 1983) was used to measure pregnant women's state anxiety. The STAI-S is a 20 -item selfreport questionnaire that assesses emotional, physiological, and cognitive symptoms of anxiety ( $1-$ not at all to $4-$ very much so). Sample items included "I am worried" and "I feel steady (reverse coded)". A total score was calculated by summing all items. Higher scores indicated greater state-anxiety (Cronbach's $\alpha=.95$ ).

\section{Pregnancy-specific anxiety (Table 1, e)}

Pregnant mothers reported on a 4-point scale $(1-$ never or not at all to 4 - a lot of the time or very much) about her worries and concerns about her health, her baby' health, and upcoming labor and delivery over the previous week (Rini, DunkelSchetter, Wadhwa, \& Sandman, 1999). Sample items included "I have a lot of fear regarding the health of my baby". A total score was calculated by summing all 10 items, with higher scores indicating greater state-anxiety $(\alpha=.95)$.

\section{Maltreatment history (Table 1, f)}

The Childhood Trauma Questionnaire-Short Form (CTQ-SF; Bernstein et al., 2003) was used to assess possibility of maltreatment from birth to age 18. Participants reported on a 5-point scale $(1$ - never true to 5 - very often true) for 28 items. Sample items included "I felt that someone in my family hated me" and "I was punished with a belt, a board, or a cord (or some other hard object)". A total score was calculated by summing all items, with higher scores indicating more severe experiences $(\alpha=.87)$. Due to the high skewness (skewness $=1.54)$ of the raw total score, a log-transformed total score was used in final analysis.

\section{Hair cortisol (Table 1, g)}

Following established procedures (D'Anna-Hernandez, Ross, Natvig, \& Laudenslager, 2011), women's hair samples were collected from the posterior vertex region and as close to the scalp as possible by trained experimenters from a $1 \mathrm{~cm}^{2}$ patch using hair cutting shears. The scalp end was marked, and the hair samples were wrapped in aluminum foil and stored in a small plastic tube at room temperature until shipment to the laboratory in Dresden, Germany, for analysis. Cortisol was extracted from whole, nonpulverized hair and analyzed by a Shimadzu HPLC-tandem mass spectrometry system (Shimadzu, Canby, OR) coupled to an ABSciex API 5000 Turbio-ion-spray triple quadrupole tandem mass spectrometer (AB Sciex, Foster City, CA; Gao et al., 2013).

In our study, a $6 \mathrm{~cm}$ length of hair was sampled to approximate hair cortisol concentrations during the previous 6 months of pregnancy, which was also the length of time during which women reported on their chronic and episodic life stress levels. The sensitivity, specificity, and reliability are well established (intra and inter-assay CVs between 3.7 and 8.8\%; Gao et al., 2013).

\section{Economic hardship (Table 1, h)}

The Economic Hardship Questionnaire (EHQ; Barrera, Caples, \& Tein, 2001) was used to assess four areas of economic hardship, including how much financial strain they felt, their ability to make ends meet, having enough money for necessities, and whether they had to make any lifestyle adjustments due to 
financial needs. A total score was calculated by averaging all four subscales' weighted $z$ scores (Barrera et al., 2001), with higher scores indicating greater economic hardship $(\alpha=.93)$.

\section{Family resources (Table 1, i)}

The Family Resource Scale (Dunst \& Leet, 1987) was used. Participants rated on a 5-point scale $(1-$ not at all adequate to 5 - almost always adequate) about family concerns and priorities regarding family needs, such as growth/support, health/necessities, physical necessities, physical shelter, intra-family support, communication/employment, childcare, and personal resources. A total score was calculated by averaging all 30 items, with a higher score indicating more adequate family resources $(\alpha=.94)$.

\section{Day-to-day stress (Table 1, j)}

The Everyday Stress Index (ESI; Hall, 1990) was used to assesses the extent to which participants were bothered by daily problems from different areas of life. These areas included role overload, financial concerns, parenting worries, employment problems, and interpersonal conflict. Participants rated on a 4-point scale $(0$-not at all bothered to 3 - bothered a great deal) for 30 items. A total score was calculated by summing all 30 items, with a higher score indicating more everyday stress $(\alpha=.89)$.

\section{Affect intensity (Table 1, k)}

The magnitude or intensity of participants' emotions were measured with the Affect Intensity Measure (AIM; Larsen \& Diener, 1987). A shortened 20-item version of the questionnaire was used; sample items included "When I'm happy, I feel like I'm bursting with joy". A total score was calculated by averaging all items, with a higher score indicating more intense affect $(\alpha$ $=.76)$.

\section{Impulsivity (Table 1, I)}

The urgency subscale of the Urgency, Premeditation, Perseverance, Sensation seeking, Impulsive behavior scale (UPPS; Whiteside \& Lynam, 2001) was used. Participants rated on a 4-point scale (1 - agree strongly to 4 - disagree strongly) for 12 items. Sample items included "I have trouble controlling my impulses". A total score was calculated by averaging all items, with a higher score indicating more impulsive tendency $(\alpha=.91)$.

\section{Mindfulness (Table 1, m)}

The Mindful Attention and Awareness Scale (MAAS; Brown \& Ryan, 2003) was used to assess the level of mindfulness. Participants rated on a 6-point $(1$ - almost always to 6 - almost never) scale about how often each statement applied to them. Sample items included "I tend not to notice feelings of physical tension or discomfort until they really grab my attention". A total score was calculated by averaging all items, with a higher score indicating higher levels of mindfulness $(\alpha=.90)$.

\section{Difficulties in accessing effective emotion regulation strategies}

(Table 1, n)

The strategy subscale of Difficulties in Emotion Regulation Scale (DERS; Gratz \& Roemer, 2004) was used to assess participants' lack of emotion regulation strategies. Participants rated on a 5-point scale ( 1 - almost never, 5 - almost always) about how often each of the 8 items applied to them. Sample item included "When I'm upset, I feel like there is nothing I can do to make myself feel better". A total score was calculated by averaging all items, with a higher score indicating more limited access to emotion regulation strategies $(\alpha=.93)$.

\section{Newborn neurobehavior}

The NICU NNNS (Lester et al., 2004) was used to assess newborn neurobehavior. Infant behavior was assessed shortly after birth with 13 summary scales, including self-regulation, amount of handling, habituation, stress signs, arousal, excitability, lethargy, nonoptimal reflexes, asymmetrical reflexes, hyper and hypotonicity, and quality of movement in the newborn. Two factor scores Arousal and Attention - were derived from the summary scales. Arousal comprised a higher handling score, decreased selfregulation, and more stress, arousal, and excitability signs $(\alpha=$ $0.84)$. Attention comprised high attention scores and low lethargy scores $(\alpha=0.85)$. Our NNNS assessment, procedures, and factor analyses were described elsewhere (Ostlund et al., 2019).

\section{Data analyses}

We conducted multigroup path analyses, which allowed simultaneous estimation of path coefficients for male and female newborns in one model and tests of sex differences in these path coefficients. For each RDoC construct, we fit two multigroup path models (an $a$ model and a $b$ model) sequentially. In $a$ models, we allowed all paths to vary across the two sex groups; in $b$ models, we constrained the paths to be equal across groups. A significantly better model fit for $a$ model than its corresponding $b$ model would indicate that the paths differ between males and females. The comparison was achieved by comparing changes in $\chi^{2}$ values and degrees of freedom. Five sets of multigroup path models were examined: Model $1 \mathrm{a}$ and $1 \mathrm{~b}$ for acute threat, Model $2 \mathrm{a}$ and $2 \mathrm{~b}$ for potential threat, Model $3 \mathrm{a}$ and $3 \mathrm{~b}$ for sustained threat, Model 4a and $4 \mathrm{~b}$ for arousal, and Model 5a and $5 \mathrm{~b}$ for regulation. All models were conducted using Mplus Version 7 (Muthén \& Muthén, 1998-2015). Missing data were handled with robust maximum likelihood estimation with standard errors. Model fit was evaluated with multiple fit indices, including $\chi^{2}$, root mean square error of approximation (RMSEA), comparative fit index (CFI), and standardized root mean square residual (SRMR). Nonsignificant $\chi^{2}$, RMSEA < 0.06 , CFI $>0.95$, and SRMR $<0.08$ would indicate good model fit (Hu \& Bentler, 1999).

\section{Results}

Means and standard deviations for the measures included in the models are displayed in Table 2, organized by infant sex. A bivariate correlation matrix among all study variables for infant boys and girls are provided in Supplementary Table S1 (supplementary material). Following our preregistered analytical approach, multigroup path models were applied. Household income was controlled in all path models for its association with newborn attention. As shown in Table 3, the models that allowed all paths to vary between male and female groups (Models 1a-4a) were not statistically different from the models that constrained the paths to be equal across males and females (Models 1b-4b), with the exception of nested Model $5 \mathrm{a}$ and 5b. According to the parsimonious principle of model selection, we would select the constrained models (see Supplementary Figure S1, available online, for results from these models). However, we chose the free-to-vary models for three reasons. 
Table 2. Descriptive statistics for all study variables by infant sex

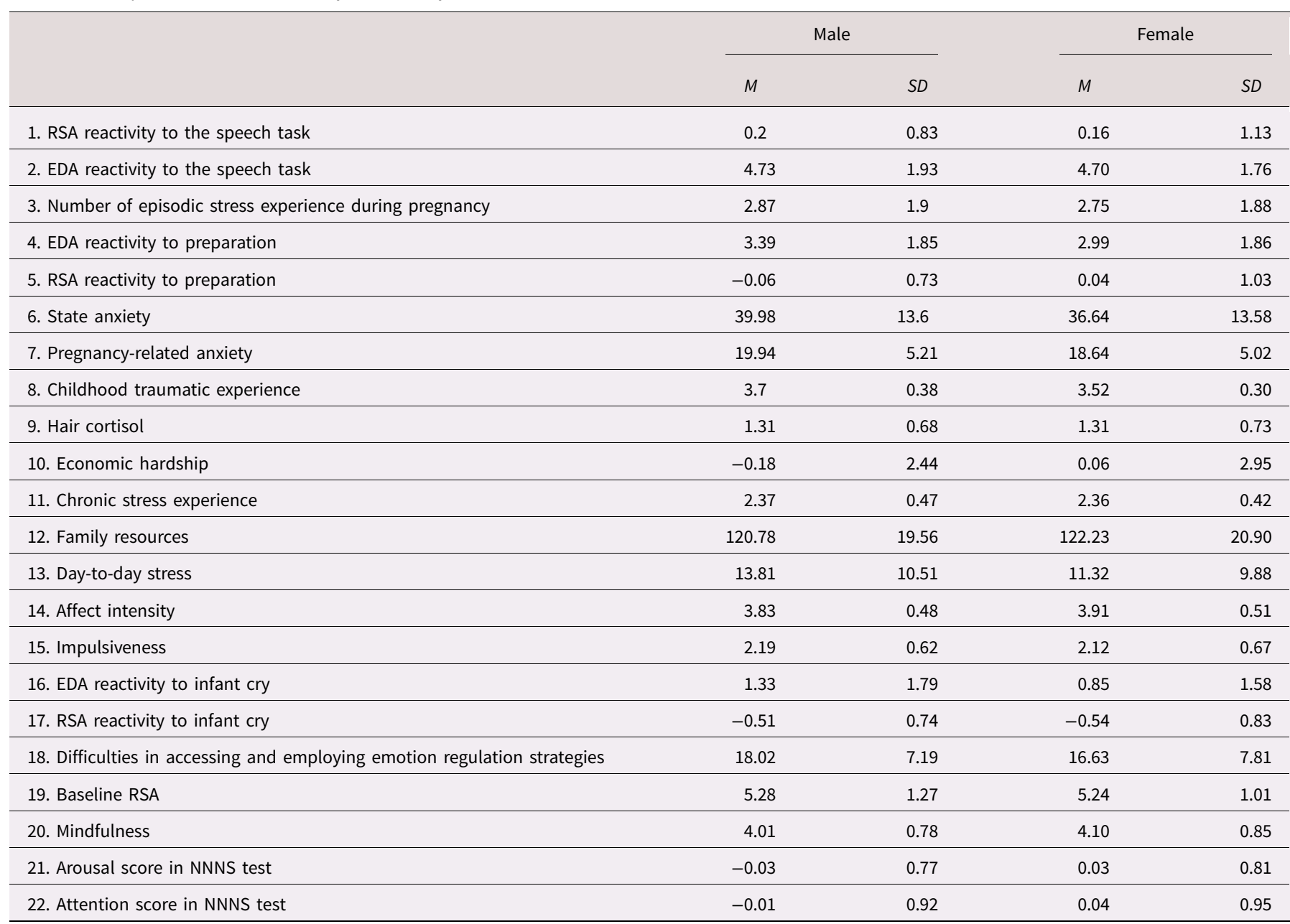

EDA = electrodermal reactivity; NNNS = NICU Network Neurobehavioral Scale; RSA = respiratory sinus arrhythmia

First, as presented in Table 3, although $\chi^{2}$ statistics of the constrained models indicated good fit (i.e., nonsignificant $\chi^{2}$ ), other fit indices indicated otherwise. Specifically, all constrained models had poor CFI (i.e., CFIs < .95) and most of them had poor RMSEA and SRMR (Hu \& Bentler, 1999). Second, males and females exhibited distinct patterns of results, as indicated by the different magnitude, sometimes even signs, of the standardized coefficients in the free-to-vary models (see Figure 1). We speculated that our relatively small sample may have limited power in detecting meaningful sex differences in these models. Finally, our decision was rooted in a well-established, theoretically informed, and continuously growing body of literature documenting sex differences in prenatal programming (Sandman, Glynn, \& Davis, 2013). Taken together, we decided to report results obtained from models where effects were allowed to differ for male and female newborns.

\section{Negative valence systems and newborn neurobehavior}

Figures $1 \mathrm{a}, 1 \mathrm{~b}$, and $1 \mathrm{c}$ present estimated path coefficients for the three models that examined Negative Valence Systems constructs. In general, as hypothesized, markers of prenatal stress had different effects on female and male newborns' neurobehavioral outcomes. For the acute threat model (Figure 1a), women who experienced more episodic stress during pregnancy tended to have male, but not female, infants who were less aroused during the NNNS exam $(\beta=-.31, p<.01)$. In contrast, more episodic stress experiences during pregnancy predicted low NNNS attention scores for female, but not male, newborns' $(\beta=-.30, p<.05)$. In the potential threat model (Figure 1b), women who reported high levels of pregnancy-specific anxiety had male newborns with high NNNS attention $(\beta=.31, p<.05)$. In the sustained threat model (Figure 1c), high levels of economic hardship $(\beta=.47, p<.01)$ and low levels of perceived everyday stress $(\beta=-.36, p<.05)$ predicted high NNNS attention for female newborns.

\section{Arousal/regulatory systems and newborn neurobehavior}

None of the indicators we identified for arousal predicted newborn neurobehavior (see Figure 1d). In the regulation model (Figure 1e), high levels of maternal mindfulness predicted high NNNS arousal among female newborns $(\beta=.31, p<.05)$. In addition, high prenatal baseline RSA $(\beta=.19, p<.05)$ and greater difficulty accessing emotion regulation strategies $(\beta=.44, p<.001)$ predicted low NNNS attention among only female newborns.

\section{Discussion}

This study characterized the complex construct of prenatal stress across physiological, psychological, and behavioral levels within 
Table 3. Fit indices for nested sequences of model tests

\begin{tabular}{|c|c|c|c|c|c|c|c|c|c|}
\hline Model & $\chi^{2}$ & $d f$ & $c$ & RMSEA & $\mathrm{CFI}$ & SRMR & $c d$ & $\chi^{2}$ diff & $\Delta d f$ \\
\hline \multicolumn{10}{|l|}{ Aim 1} \\
\hline \multicolumn{10}{|l|}{ Acute Threat } \\
\hline Model 1a & 0.1 & 2 & 1.16 & 0 & 1 & 0.005 & & & \\
\hline Model $1 \mathrm{~b}$ & 14.03 & 10 & 0.88 & 0.077 & 0.72 & 0.057 & 0.81 & 15.06 & 8 \\
\hline \multicolumn{10}{|c|}{ Potential Threat } \\
\hline Model 2a & 2.14 & 2 & 1.02 & 0.035 & 0.983 & 0.022 & & & \\
\hline Model $2 b$ & 17.2 & 12 & 1.00 & 0.089 & 0.372 & 0.067 & 1.00 & 15.08 & 10 \\
\hline \multicolumn{10}{|c|}{ Sustained Threat } \\
\hline Model 3a & 0.06 & 2 & 0.99 & 0 & 1 & 0.002 & & & \\
\hline Model 3b & 16.2 & 16 & 1.03 & 0.015 & 0 & 0.042 & 1.04 & 16.05 & 14 \\
\hline \multicolumn{10}{|l|}{ Aim 2} \\
\hline \multicolumn{10}{|l|}{ Arousal } \\
\hline Model 4a & 0.08 & 2 & 1.34 & 0 & 1 & 0.005 & & & \\
\hline Model 4b & 13.19 & 12 & 1.05 & 0.038 & 0.63 & 0.054 & 0.99 & 13.86 & 10 \\
\hline \multicolumn{10}{|l|}{ Regulatory } \\
\hline Model 5a & 0.07 & 2 & 1.48 & 0 & 1 & 0.005 & & & \\
\hline Model 5b & $19.50^{*}$ & 10 & 1.06 & 0.114 & 0.618 & 0.082 & 0.96 & $21.51^{\star}$ & 8 \\
\hline
\end{tabular}

Note. ${ }^{\star} p<.05$.

$c=$ weighting constant for computing the $\chi^{2}$ statistic using robust estimation method; $c d=$ weighting constant for the difference between two $\chi^{2}$ values using robust estimation. RMSEA $=$ root mean square error of approximation; CFI = comparative fit index; SRMR = standardized root mean square residual. $\chi^{2}$ difference value $\left(\chi^{2}\right.$ diff $)$ is computed by incorporating the difference test scaling correction, following Satorra-Bentler scaled $\chi^{2}$ difference test.

the RDoC framework (Doyle \& Cicchetti, 2018). We identified several RDoC-informed indices of prenatal stress that predicted distinct patterns of newborn neurobehavior, particularly attention in female newborns. Our findings add specificity to the influences of prenatal stress indicators on newborn neurobehavior and may inform understanding of the neurodevelopmental origins of psychopathology.

Within the RDoC-Negative Valence Systems domain, we found that high maternal episodic stress and perceived daily stress, but lower economic hardship, were related to low attention in female newborns. As for male newborns, high episodic stress was related to low arousal and high pregnancy-specific anxiety was related to high attention. These results replicate previous findings of altered fetal and newborn outcomes, suggestive of a programming effect among infants with high prenatal maternal stress (Bryant Borders et al., 2007; Ostlund et al., 2019). Surprisingly, no physiological marker under this domain predicted newborn attention or arousal. Rather, expectant mothers' subjective experience (e.g., episodic stress experiences) seemed to have a more pronounced influences on newborn neurobehavior relative to indicators of their peripheral physiology, highlighting the importance of women's perceptions of prenatal stress. Another possible explanation for the lack of an association between physiological marker and newborn neurobehavior may be the task that was used to elicit physiological reactivity. RSA reactivity induced by the highly structured TSST may not have accurately captured pregnant women's physiological response to the stressful events in their everyday lives. On the other hand, baseline RSA, which is more reflective of women's general regulatory capacities, was associated with female newborns' attention. Altogether, these findings highlight the need for future studies to incorporate both subjective and physiological indices when studying prenatal stress under the RDoC framework (Cicchetti, 2008; Doyle \& Cicchetti, 2018).

Different risk markers within the Negative Valence Systems domain seemed to have distinct implications for neonatal attention. Although high maternal episodic stress and perceived daily stress predicted low neonatal attention in female newborns, high economic stress predicted high attention in female newborns and high pregnancy-specific anxiety predicted high attention in male newborns. We speculate that these distinct associations may reflect unique patterns of fetal adaptation based on varied maternal experiences. Prenatal programming theories posit that intrauterine experiences shape fetal neurodevelopmental systems, with implications for mental and physical health across the life span (Van den Bergh, 2011). Mothers' experiences of stress during pregnancy are thought to provide ongoing signals to the developing fetus about postnatal conditions, which affects the fetus' neurobehavioral development in a manner that best promotes newborn survival in the immediate extrauterine environment, potentially at the expense of long-term health. On one hand, newborns whose mothers had high pregnancy-specific anxiety and economic stress may be more vigilant and attentive, such that they would better engage with the environment to obtain resources (i.e., greater attention). On the other hand, when maternal distress is characterized by episodic and daily perceived stress, infants may show blunted neurobehavior (i.e., inattention) as a means of adapting to a potentially chaotic and risky environment. To fully address and interpret the influence of each prenatal maternal stress and newborn neurobehavior, future research assessing infant long-term developmental outcomes is essential. 
(a)

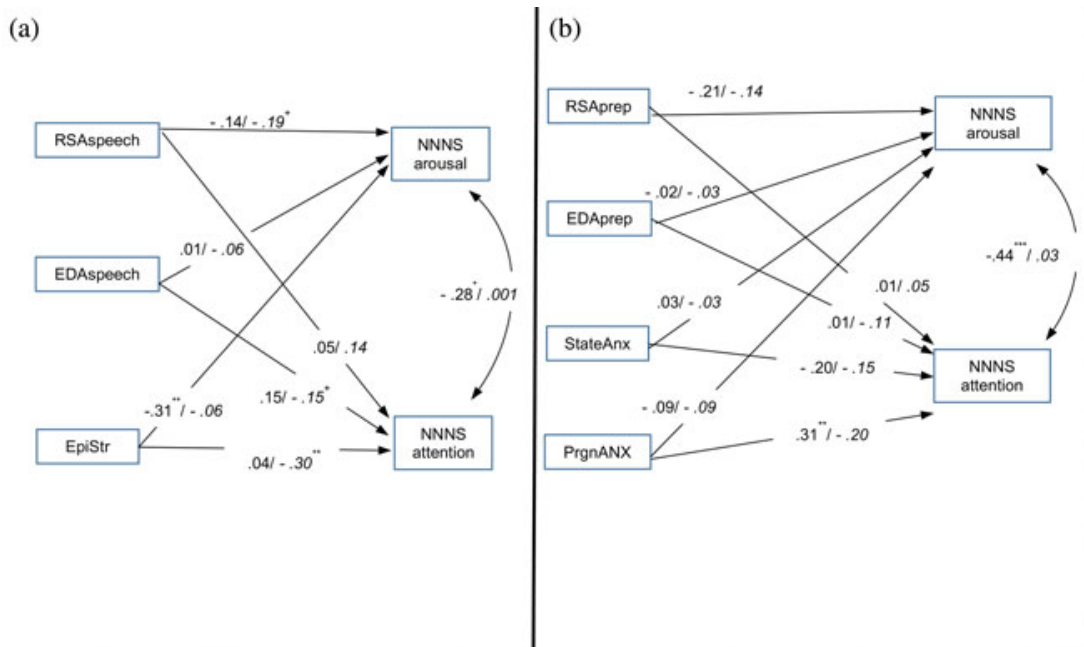

(c)

(d)
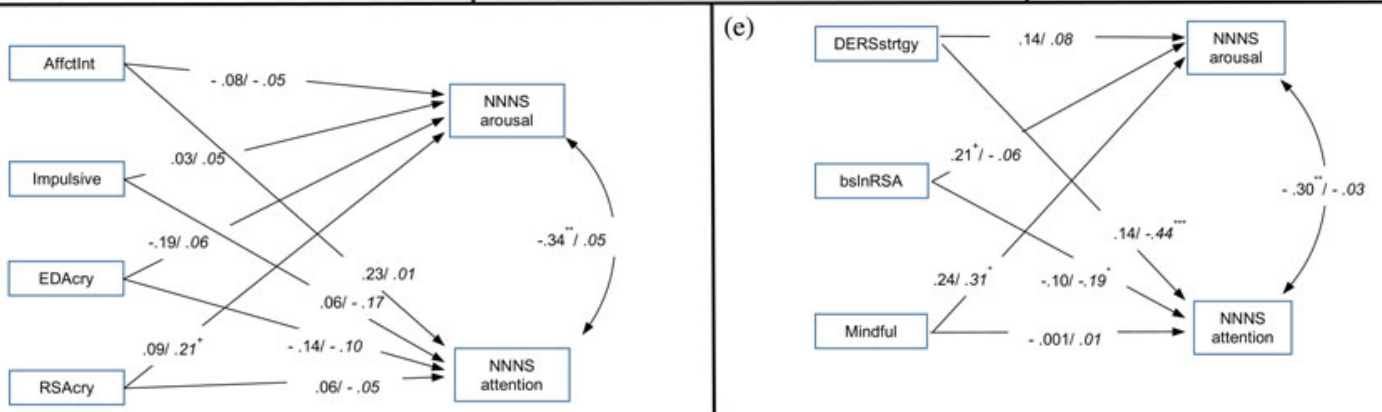

Figure 1. Multigroup path models testing the role of acute threat (1a), potential threat (1b), sustained threat (1c), arousal (1d), and regulatory (1e) Research Domain Criteria (RDoC) domains in predicting newborn neurobehavior. Path coefficients and covariations before the slash (/) reflect estimates for male infants, whereas italicized figures after the slash (/) reflect estimates for female infants. RSAspeech = respiratory sinus arrhythmia (RSA) reactivity to the speech task; EDAspeech = and electrodermal $(E D A)$ reactivity to the speech task; EpiStrs = number of episodic stress experience during pregnancy; EDAprep = EDA reactivity to preparation; RSAprep = RSA reactivity to preparation; StateAnx = state anxiety; PrgnAnx = Pregnancy-related anxiety; ChildTrauma = childhood traumatic experience; HairCort = hair cortisol; EcoHard = economic hardship; ChrStrs = chronic stress experience; FamRsrc = family resources; DailyStr = day-to-day stress; AffctInt = affect intensity; Impulsive = impulsiveness; EDAcry = EDA reactivity to baby's cry; RSAcry = RSA reactivity to babies' cry; DERSstrtgy = difficulties in accessing and employing emotion regulation strategies; bsInRSA = baseline RSA; Mindful = mindfulness; NNNS = NICU Network Neurobehavioral Scale (NNNS) arousal = arousal score in NNNS test; NNNS attention $=$ attention score in NNNS test. ${ }^{*} p<.05,{ }^{\star \star} p<.01,{ }^{\star} p<.001$.

Within the Arousal/Regulatory Systems domain, mothers who reported more difficulties with emotion regulation strategies had female infants with lower attention, showing less alertness to the environment. In addition, female newborns whose mothers reported less dispositional mindfulness exhibited lower arousal rather than higher arousal as we had hypothesized - indicated by less sensitivity to the environment, higher threshold for stimulation, and a lower proneness to excitability. Taken together, in our sample, signs of maternal regulatory difficulties during pregnancy were related to a blunted pattern of reactivity to the environment in female newborns. However, whether this blunted pattern confers risk for later child outcomes or reflects adaptivity to the environment remains to be empirically examined. Although high newborn arousal has been linked to childhood psychopathology, it could also be adaptive because highly aroused neonates may be more effective in obtaining resources from the caregiver or the environment. In this case, promoting prenatal maternal regulatory abilities, such as effective emotion regulation strategies and mindfulness, could foster newborns' engagement with the environment and subsequently lead to beneficial developmental outcome.
Contrary to these self-report measures, physiological regulation (indexed by baseline RSA) was associated with female newborns' attention in the opposite direction: high maternal baseline RSA predicted female newborns' low attention scores, which was inconsistent with our hypothesis. This finding is particularly interesting, considering that maternal baseline RSA did associate with other self-report measures of maternal regulation in expected directions, such that low maternal baseline RSA was related to difficulty in accessing emotion regulation strategies and low mindfulness. We speculate that physiological and behavioral markers of maternal regulation prenatally may program different neurobehavioral profiles. High baseline RSA, indicating greater inhibitory influence on cardiac output (Beauchaine, 2015), may send a signal to the fetus that an extrauterine environment is safe and does not demand an alerted state. Resultantly, newborns of mothers with high RSA tend to show less attention than those of low-RSA mothers. High levels of behavioral regulation, on the other hand, may signal an extrauterine environment that requires more regulatory efforts; therefore, newborns of more mindful mothers may show high levels of attention, possibly as a 
way to stay alert. This speculation remains to be further evaluated by future research.

In our study, female appeared to be more vulnerable than male newborns to RDoC-informed prenatal stress, manifested by more significant predictive associations between prenatal maternal stress and newborn neurobehavior for females than for males. This finding was contrary to our hypothesis built upon accumulating evidence that male fetuses exposed to prenatal adversities tended to endure more impaired developmental outcomes (Dipietro \& Voegtline, 2017). However, this is not the first study to report evidence of female vulnerability. For example, after reanalyzing numerous studies on sex differences from their team, Sandman and colleagues found that female offspring were more influenced by exposure to early adversities, especially with regard to affective outcomes (Sandman et al., 2013). In addition, the focal newborn outcome in our study - neurobehavior - is different from medical outcomes (e.g., preterm birth and perinatal morbidities) in the existing literature that supports the male vulnerability hypothesis. Therefore, we could not discern whether the female vulnerability found in our study was specific to neurobehavioral functions. Future studies that assess multiple domains of newborn functions are needed in order to make firmer interpretations of our current findings.

This study has numerous strengths. First, the aims and hypotheses were preregistered prior to study analyses. By participating in open and transparent science, we hope to contribute to replicability and reproducibility of findings. Second, we employed a standardized protocol to assess newborn neurobehavior within hours after birth for most participants, which allowed us to limit potential confounders of parent-child interactions and detect the direct influence of prenatal maternal $\mathrm{RDoC}$-informed risk on the offspring. Given that NNNS scores are considered a tool for identifying high-risk newborns, linking prenatal RDoC markers to the NNNS further facilitates early identification of psychopathology and informs early intervention efforts. Third, we collected extensive data on prenatal stress that ranged from contextual, behavioral, emotional, and physiological levels. This rich information not only facilitated a comprehensive characterization of women's experiences during pregnancy, but advanced identification of multiple markers of prenatal maternal risk under the RDoC framework.

Our study was not without limitations. First, some markers of prenatal stress in our study included stressors in women's early life, such as maltreatment history, and in different stages of pregnancy, such as episodic stress experienced in the past 6 months. Therefore, we could not examine whether the timing of exposure to prenatal stress was related to infant neurobehavioral outcomes (Liu et al., 2010). In addition, because the mother-child dyads were biologically related, we could not rule out potential genetic effects that may account for intergenerational risk transmission. Lastly, despite our efforts to recruit women from minority racial and ethnic groups, our current sample was mostly White, which suggests caution when generalizing our findings.

By extending the RDoC framework to the perinatal periods, this study provided initial support that the $\mathrm{RDoC}$ is a useful framework for investigating the complexities of prenatal stress and its heterogenous effects on neonatal neurobehavioral competencies. Because newborn neurobehavior has been found to predict elevated psychopathology risk in childhood (Liu et al., 2010), early identification may facilitate targeted preventive interventions to avert children from developmental trajectories of psychopathology. Furthermore, by identifying specific RDoC-informed, prenatal stress indicators newborn neurobehavior, our findings suggest that interventions may be implemented even earlier in life - during pregnancy - to ameliorate risk for childhood psychopathology.

Supplementary Material. The Supplementary Material for this article can be found at https://doi.org/10.1017/S0954579420002266.

Acknowledgments. The authors would like to thank all of the families who generously donated their time to participate in our study. We would also like to thank Mike Varner and Bob Silver for their support of the BABY study and for providing their dedicated OBGYN Research Network staff to help with screening and recruitment. We thank Connie Hammen for her assistance with training on the UCLA Life Stress Interview. We would also like to thank the University of Utah Vice President's Clinical Translational Research Scholars program for their mentorship and grantsmanship assistance. Last but not the least, we thank Celine Saenz and Sarah Terrell for their hard work on recruitment, coordination, and study management.

Funding Statement. This work was funded by the National Institute of Mental Health grants R01MH119070 and R21MH109777 (S.C. and E.C.), the National Institute on Drug Abuse career development award 7K08DA038959-02 (E.C.), and grants from the University of Utah Consortium for Families and Health Research and Interdisciplinary Research Pilot Program.

Conflict of Interest. None.

\section{References}

Ablow, J. C., Marks, A. K., Feldman, S. S., \& Huffman, L. C. (2013). Associations between first-time expectant women's representations of attachment and their physiological reactivity to infant cry. Child Development, 84, 1373-1391. doi:10.1111/cdev.12135

Arns, M., Swatzyna, R. J., Gunkelman, J., \& Olbrich, S. (2015). Sleep maintenance, spindling excessive beta and impulse control: An RDoC arousal and regulatory systems approach? Neuropsychiatric Electrophysiology, 1, 1-11. doi:10.1186/s40810-015-0005-9

Barrera, M., Caples, H., \& Tein, J. Y. (2001). The psychological sense of economic hardship: Measurement models, validity, and cross-ethnic equivalence for urban families. American Journal of Community Psychology, 29, 493-517. doi:10.1023/A:1010328115110

Beauchaine, T. P. (2015). Respiratory sinus arrhythmia: A transdiagnostic biomarker of emotion dysregulation and psychopathology. Current Opinion in Psychology, 3, 43-47. doi:10.1016/j.copsyc.2015.01.017

Bernstein, D. P., Stein, J. a., Newcomb, M. D., Walker, E., Pogge, D., Ahluvalia, T., ... Zule, W. (2003). Development and validation of a brief screening version of the childhood trauma questionnaire. Child Abuse \& Neglect, 27, 169-190. doi:10.1016/S0145-2134(02)00541-0

Braeken, M. A. K., Jones, A., Otte, R. A., Widjaja, D., Huffel, S. V., Monsieur, G. J. Y. J., ... Bergh, B. R. H. V. D. (2015). Anxious women do not show the expected decrease in cardiovascular stress responsiveness as pregnancy advances. Biological Psychology, 111, 83-89. doi:10.1016/j.biopsycho.2015.08.007

Brown, K. W., \& Ryan, R. M. (2003). The benefits of being present: Mindfulness and its role in psychological well-being. Journal of Personality and Social Psychology, 84, 822-848. doi:10.1037/0022-3514.84.4.822

Bryant Borders, A. E., Grobman, W., Amsden, L. B., \& Holl, J. L. (2007). Chronic stress and low birth weight neonates in a low-income population of women. Obstetrics \& Gynecology, 109, 331-338. doi:10.1097/ 01.AOG.0000250535.97920.b5

Buss, C., Entringer, S., Moog, N. K., Toepfer, P., Fair, D. A., Simhan, H. N., ... Wadhwa, P. D. (2017). Intergenerational transmission of maternal childhood maltreatment exposure: Implications for fetal brain development. Journal of the American Academy of Child \& Adolescent Psychiatry, 56, 373-382. doi:10.1016/j.jaac.2017.03.001

Cicchetti, D. (2008). A multiple-levels-of-analysis perspective on research in development and psychopathology. In T. P. Beauchaine \& S. P. Hinshaw (Eds.), Child and adolescent psychopathology (pp. 27-57). Hoboken, NJ: Wiley. 
D’Anna-Hernandez, K. L., Ross, R. G., Natvig, C. L., \& Laudenslager, M. L. (2011). Hair cortisol levels as a retrospective marker of hypothalamicpituitary axis activity throughout pregnancy: Comparison to salivary cortisol. Physiology and Behavior, 104, 348-353. doi:10.1016/j.physbeh.2011.02.041

Dawson, M. E., Schell, A. M., \& Filion, D. L. (2007). The electrodermal system. In J. T. Cacioppo, L. G. Tassinary \& G. G. Berntson (Eds.), Handbook of psychophysiology (3rd ed., pp. 159-181). Cambridge UK: Cambridge University Press.

Dipietro, J. A., Ghera, M. M., \& Costigan, K. A. (2008). Prenatal origins of temperamental reactivity. Early Human Development, 84, 569-575. doi:10.1016/j.earlhumdev.2008.01.004.Prenatal

Dipietro, J. A., \& Voegtline, K. M. (2017). The gestational foundation of sex differences in development and vulnerability. Neuroscience, 342, 4-20. doi:10.1016/j.neuroscience.2015.07.068

Doyle, C., \& Cicchetti, D. (2018). Future directions in prenatal stress research: Challenges and opportunities related to advancing our understanding of prenatal developmental origins of risk for psychopathology. Development and Psychopathology, 30, 721-724. doi:10.1017/ S095457941800069X

Dunkel Schetter, C. (2011). Psychological science on pregnancy: Stress processes, biopsychosocial models, and emerging research issues. Annual Review of Psychology, 62, 531-558. doi:10.1146/annurev.psych.031809.130727

Dunst, C. J., \& Leet, H. E. (1987). Measuring the adequacy of resources in households with young children. Child: Care, Health and Development, 13, 111-125. doi:10.1111/j.1365-2214.1987.tb00528.x

Fernandez, K. C., Jazaieri, H., \& Gross, J. J. (2016). Emotion regulation: A transdiagnostic perspective on a New RDoC domain. Cognitive Therapy and Research, 40, 426-440. doi:10.1007/s10608-016-9772-2

Gao, W., Stalder, T., Foley, P., Rauh, M., Deng, H., \& Kirschbaum, C. (2013). Quantitative analysis of steroid hormones in human hair using a column-switching LC-APCI-MS/MS assay. Journal of Chromatography B: Analytical Technologies in the Biomedical and Life Sciences, 928, 1-8. doi:10.1016/j.jchromb.2013.03.008

Goodman, S. H., \& Gotlib, I. H. (1999). Risk for psychopathology in the children of depressed mothers: A developmental model for understanding mechanisms of transmission. Psychological Review, 106, 458-490. doi:10.1037/0033-295x.106.3.458

Gratz, K. L., \& Roemer, L. (2004). Multidimensional assessment of emotion regulation and dysregulation: Development, factor structure, and initial validation of the difficulties in emotion regulation scale. Journal of Psychopathology and Behavioral Assessment, 26, 41-54. doi:10.1023/B: JOBA.0000007455.08539.94

Hall, L. A. (1990). Prevalence and correlates of depressive symptoms in mothers of young children. Public Health Nursing, 7, 71-79. doi:10.1111/ j.1525-1446.1990.tb00615.x

Hammen, C., Adrian, C., Gordon, D., Burge, D., Jaenicke, C., \& Hiroto, D. (1987). Children of depressed mothers: Maternal strain and symptom predictors of dysfunction. Journal of Abnormal Psychology, 96, 190-198. doi:10.1037/0021-843X.96.3.190

Hu, L. T., \& Bentler, P. M. (1999). Cutoff criteria for fit indexes in covariance structure analysis: Conventional criteria versus new alternatives. Structural Equation Modeling, 6, 1-55. doi:10.1080/10705519909540118

Insel, T., Cuthbert, B., Garvey, M., Pine, D. S., Quinn, K., Sanislow, C., \& Wang, P. (2010). Research domain criteria (RDoc): Toward a new classification framework for research on mental disorders. American Journal of Psychiatry, 167(7), 748-751. doi:10.1176/appi.ajp.2010.09091379

Kirschbaum, C., Pirke, K.-M., \& Hellhammer, D. H. (1993). The 'trier social stress test'- A tool for investigating psychological stress responses in a laboratory setting. Neuropsychobiology, 28, 76-81. doi:10.1159/000119004

Kozak, M. J., \& Cuthbert, B. N. (2016). The NIMH research domain criteria initiative: Background, issues, and pragmatics. Psychophysiology, 53, 286297. doi:10.1111/psyp.12518

Larsen, R. J., \& Diener, E. (1987). Affect intensity as an individual difference characteristic: A review. Journal of Research in Personality, 21, 1-39. doi:10.1016/0092-6566(87)90023-7

Lester, B. M., \& Tronick, E. Z. (2004). History and description of the neonatal intensive care unit network neurobehavioral scale. Pediatrics, $113,634-640$.
Lester, B. M., Tronick, E. Z., \& Brazelton, T. B. (2004). The neonatal intensive care unit network neurobehavioral scale procudres.PDF. Pediatrics, 113, 641-667.

Lin, B., Kaliush, P. R., Conradt, E., Terrell, S., Neff, D., Allen, A. K., ... Crowell, S. E. (2019). Intergenerational transmission of emotion dysregulation: Part I. Psychopathology, self-injury, and parasympathetic responsivity among pregnant women. Development and Psychopathology, 31, 817-831. doi:10.1017/S0954579419000336

Liu, J., Bann, C., Lester, B., Tronick, E., Das, A., Lagasse, L., ... Bada, H. (2010). Neonatal neurobehavior predicts medical and behavioral outcome. Pediatrics, 125, doi:10.1542/peds.2009-0204

Loomans, E. M., Van Dijk, A. E., Vrijkotte, T. G. M., Van Eijsden, M., Stronks, K., Gemke, R. J. B. J., \& Van Den Bergh, B. R. H. (2013). Psychosocial stress during pregnancy is related to adverse birth outcomes: Results from a large multi-ethnic community-based birth cohort. European Journal of Public Health, 23, 485-491. doi:10.1093/eurpub/cks097

Mittal, V. A., \& Wakschlag, L. S. (2017). Research domain criteria (RDoC) grows up: Strengthening neurodevelopment investigation within the RDoC framework. Journal of Affective Disorders, 216, 30-35. doi:10.1016/ j.jad.2016.12.011

Monk, C., \& Hane, A. A. (2014). Fetal and infant neurobehavioral development: Basic processes and environmental influences. In A. Wenzel (Ed.), The Oxford handbook of perinatal psychology (pp. 1-60). New York: Oxford University Press. doi:10.1093/oxfordhb/9780199778072.013.20

Muthén, L. K., \& Muthén, B. O. (1998-2015). Mplus user's guide (7th ed.). Los Angeles, CA: Muthén \& Muthén.

O’Donnell, K. J., Glover, V., Barker, E. D., \& O'Connor, T. G. (2014). The persisting effect of maternal mood in pregnancy on childhood psychopathology. Development and Psychopathology, 26, 393-403. doi:10.1017/ S0954579414000029

Orta, O. R., Tworoger, S. S., Terry, K. L., Coull, B. A., Gelaye, B., Kirschbaum, C., ... Williams, M. A. (2019). Stress and hair cortisol concentrations from preconception to the third trimester. Stress, 22, 60-69. doi:10.1080/ 10253890.2018.1504917

Ostlund, B. D., Vlisides-Henry, R. D., Crowell, S. E., Raby, K. L., Terrell, S., Brown, M. A., ... Conradt, E. (2019). Intergenerational transmission of emotion dysregulation: Part II. Developmental origins of newborn neurobehavior. Development and Psychopathology, 833-846. doi:10.1017/ S0954579419000440

Porges, S. W. (2007). The polyvagal perspective. Biological Psychology, 74, 116-143. doi:10.1016/j.biopsycho.2006.06.009

Rini, C. K., Dunkel-Schetter, C., Wadhwa, P. D., \& Sandman, C. A. (1999). Psychological adaptation and birth outcomes: The role of personal resources, stress, and sociocultural context in pregnancy. Health Psychology, 18, 333-345. doi:10.1037/0278-6133.18.4.333

Robinson, M., Mattes, E., Oddy, W. H., Pennell, C. E., Eekelen, A. V. A. N., Mclean, N. J., ... Newnham, J. P. (2011). Prenatal stress and risk of behavioral morbidity from age 2 to 14 years: The influence of the number, type, and timing of stressful life events. Development and Psychopathology, 23, 507-520. doi:10.1017/S0954579411000241

Sandman, C. A., Glynn, L. M., \& Davis, E. P. (2013). Is there a viability vulnerability tradeoff ? Sex differences in fetal programming. Journal of Psychosomatic Research, 75, 327-335. doi:10.1016/j.jpsychores.2013.07.009

Sarchiapone, M., Gramaglia, C., Iosue, M., Carli, V., Mandelli, L., Serretti, A., ... Zeppegno, P. (2018). The association between electrodermal activity (EDA), depression and suicidal behaviour: A systematic review and narrative synthesis. BMC Psychiatry, 18, 1-27. doi:10.1186/s12888-017-1551-4

Spielberger, C. D., Gorsuch, R. L., Lushene, R., Vagg, P. R., \& Jacobs, G. A. (1983). Manual for the state-trait anxiety inventory. Palo Alto, CA: Consulting Psychologists Press.

Spinillo, A., Montanari, L., Gardella, B., Roccio, M., Stronati, M., \& Fazzi, E. (2009). Infant sex, obstetric risk factors, and 2-year neurodevelopmental outcome among preterm infants. Developmental Medicine and Child Neurology, 51, 518-525. doi:10.1111/j.1469-8749.2009.03273.x

Van den Bergh, B. R. H. (2011). Developmental programming of early brain and behaviour development and mental health: A conceptual framework. Developmental Medicine \& Child Neurology, 53, 19-23. doi:10.1111/ j.1469-8749.2011.04057.x 
van den Heuvel, M. I., Johannes, M. A., Henrichs, J., \& Van den Bergh, B. R. H. (2015). Maternal mindfulness during pregnancy and infant socio-emotional development and temperament: The mediating role of maternal anxiety. Early Human Development, 91, 103-108. doi:10.1016/j.earlhumdev.2014.12.003

van Dooren, M., de Vries, J. J. G. G. J., \& Janssen, J. H. (2012). Emotional sweating across the body: Comparing 16 different skin conductance measurement locations. Physiology and Behavior, 106, 298-304. doi:10.1016/ j.physbeh.2012.01.020

Wachman, E. M., Hunter, R. G., Shrestha, H., Lapp, H. E., Meyer, J., Alvarez, C. D., \& Tronick, E. (2019). Maternal hair cortisol levels as a novel predictor of neonatal abstinence syndrome severity: A pilot feasibility study. Developmental Psychobiology, 62, 116-122. doi:10.1002/ dev.21896

Whiteside, S. P., \& Lynam, D. R. (2001). The five factor model and impulsivity: Using a structural model of personality to understand impulsivity. Personality and Individual Differences, 30, 669-689. doi:10.1016/S01918869(00)00064-7

Willoughby, M., Greenberg, M., Blair, C., \& Stifter, C. (2007). Neurobehavioral consequences of prenatal exposure to smoking at 6 to 8 months of age. Infancy, 12, 273-301. doi:10.1111/j.1532-7078.2007.tb00244.x 